\title{
La anticoncepción de emergencia en Chile: estructuración de su demanda en función de variables socioeconómicas
}

\author{
José Manuel Morán Faúndes*
}

En enero del año 2010 se aprobó en Chile la ley 20.418 que restableció la entrega de la anticoncepción de emergencia (píldora del día después) en el servicio público de salud del país, luego de que su distribución fuese prohibida por el Tribunal Constitucional en el 2007. A más de un año de la entrada en vigencia de la ley, el Ministerio de Salud publicó datos oficiales acerca de la entrega de este método anticonceptivo para el año 2010, lo que abre nuevas posibilidades para comprender los niveles de accesibilidad y de demanda. El presente trabajo analiza por primera vez la estructuración de dicha demanda en el Sistema Nacional de Servicios de Salud, evaluando la incidencia de tres variables socioeconómicas: el estatus económico, el nivel de educación y la zona de residencia. Los resultados muestran que la tasa media de consultas por anticoncepción de emergencia en mujeres de 15 a 44 años fue de 2,53 consultas por cada mil mujeres en edad fértil. Esta tasa llega a 4,27 en mujeres adolescentes de entre 15 y 19 años, y sólo a 2,1 en mujeres de 20 a 44. Los mayores niveles de demanda se sitúan en las comunas con indicadores socioeconómicos deficientes, esto es, alto porcentaje de población comunal con un ingreso autónomo bajo, importante proporción de personas sin educación secundaria completa, y un alto porcentaje de población residente en zonas rurales.

Palabras clave: Anticoncepción de emergencia. Píldora del día después. Chile. Reproducción. Fertilidad.

\footnotetext{
* Doctorando en Estudios Sociales de América Latina (Universidad Nacional de Córdoba), maestro en Sociología (Universidad Nacional de Córdoba), cientísta político (Pontificia Universidad Católica de Chile). Becario Consejo Nacional de Investigaciones Científicas y Técnicas - Conicet, Centro de Investigaciones Jurídicas y Sociales - Universidad Nacional de Córdoba (jmfmoran@gmail.com).
} 


\section{Introducción}

La anticoncepción de emergencia se refiere a aquellos métodos que pueden ser utilizados por la mujer en los días siguientes a haber mantenido una relación sexual con riesgo de embarazo, a fin de evitar el mismo (CROXATTO, 2004). Desde la década de los años setenta, la experimentación biomédica con altas dosis de hormonas logró desarrollar mecanismos de prevención del embarazo una vez producido el acto sexual, a través del denominado método Yuzpe (YUZPE; SMITH; RADEMAKER, 1982). Luego de una serie de investigaciones, éste método fue reemplazado por el uso de levonorgestrel solo, dados los efectos secundarios adversos del Yuzpe, y su menor efectividad en términos comparados (VON HERTZEN et al., 2002).

Los mecanismos de acción de la anticoncepción de emergencia basada en levonorgestrel han sido ampliamente estudiados, y la evidencia científica ha demostrado fundamentalmente que este método tiene efectos tanto sobre la migración espermática hacia el óvulo como sobre la ovulación, particularmente en la fase previa al momento de la fecundación. Respecto de la migración, se ha demostrado que este método anticonceptivo impide el desplazamiento de los espermatozoides alterando el espesor del moco cervical (CROXATTO, 1996), a la vez que dificulta la adhesión del espermatozoide al óvulo (DURAND et al., 2005). En lo que concierne a la ovulación, la evidencia ha demostrado que la anticoncepción de emergencia actúa además inhibiendo este proceso, lo que impide que se produzca la fecundación (SWAHN; WESTLUND; HOHANNSSON, 1996).

Sin embargo, la comunidad científica debate hoy en día respecto de un eventual tercer mecanismo de acción, que actuaría luego de producida la fecundación, esto es, la alternación del endometrio. Diversas investigaciones han buscado evidencia empírica que corrobore la acción del levonorgestrel luego de producida la unión del óvulo y el espermio, pero ésta no ha sido hallada (MENG et al, 2010). Sin embargo, esto es discutido desde posiciones que asumen que la falta de certidumbre científica sobre este punto no garantiza que la anticoncepción de emergencia no afecte necesariamente al óvulo ya fecundado (BUSQUETS, 2003; OYARZÚN, 2004).

Esta controversia no sólo tiene consecuencias dentro del mundo de la ciencia, sino que ha gatillado tensiones en otros campos y disciplinas, como el derecho y la política. Es así como, ante la sospecha de los efectos antianidatorios del levonorgestrel, diversos sectores, muchos ligados a la derecha y a la Iglesia Católica, abogan por la prohibición o penalización de su uso, mientras que otros, generalmente asociados a los movimientos feministas y de mujeres, demandan su disponibilidad y acceso en los servicios públicos de salud (MARTIN, 2004; DIDES, 2006; FAÚNDES et al., 2007; CASAS, 2009).

Este clima de tensión ha provocado una situación de alta desigualdad en América Latina respecto del acceso a la anticoncepción de emergencia. Según un informe del Consorcio Latinoamericano de Anticoncepción de Emergencia, la región de América Latina presenta casos críticos en donde se ha penalizado o prohibido su distribución en los servicios públicos 
de salud, como Costa Rica, Perú y Honduras, mientras en otros países, como México y Uruguay, la normativa vigente garantiza el acceso a prácticamente la totalidad de la población usuaria (MORÁN FAÚNDES, 2010).

Sin embargo, incluso en aquellos países donde la ley establece la entrega de anticoncepción de emergencia, diversos estudios han destacado la existencia de barreras vinculadas con la desinformación del personal médico, resistencias ideológicas o falta de recursos, todas las cuales merman la distribución y accesibilidad a este método (FIGUEIREDO et al., 2007; DE SOUZA; BRANDÃO, 2009; MORÁN FAÚNDES, 2010).

En el caso de Chile, en el año 2006 un grupo de 31 parlamentarios presentó una acción judicial ante el Tribunal Constitucional solicitando declarar inconstitucional las Normas Nacionales de Regulación de la Fertilidad, las que contemplaban la distribución de la anticoncepción de emergencia en los servicios de salud, conocida en el país como "píldora del día después". Al año siguiente, el tribunal decretó la inconstitucionalidad de la anticoncepción de emergencia, siendo ésta retirada de los servicios nacionales de salud.

A partir de ese momento, la entrega de este método desde el Estado quedó en manos de los consultorios y postas municipales, y específicamente del mandato de las y los respectivos alcaldes, quienes tenían la oportunidad de decidir autónomamente sobre su disponibilidad a nivel municipal. Las desigualdades en su entrega fueron notorias. Según una investigación realizada por FLACSO, en el 2008 sólo en el 53\% de las comunas que contaban con servicios de salud municipalizada se entregaba la anticoncepción de emergencia, cifra que disminuyó al 50,5\% en el 2009. Además, entre estas últimas, sólo un 9,3\% daba acceso a este método a cualquier mujer que lo solicitase, independientemente de los motivos que adujera, mientras que la gran mayoría restringía su accesibilidad a casos específicos (DIDES; BENAVENTE; MORÁN, 2010).

Fue recién en enero de 2010 que el gobierno de la ex presidenta Michelle Bachelet promulgó la ley 20.418 que restableció la entrega de la anticoncepción de emergencia en el servicio público de salud del país. Si bien la ley, actualmente vigente, contempla el acceso a toda mujer que lo solicite, los médicos y matronas que la entregan a menores de 14 años de edad se ven en la obligación de informar al padre, la madre o la persona adulta responsable de la solicitante, lo que genera barreras en la accesibilidad al método (COOK, 2007). Adicionalmente, la carencia de una guía técnica o protocolo oficial que reglamente la ley deja el acceso a la anticoncepción en manos del criterio de los y las médicas y matronas de turno (a excepción de los casos de violación, los que sí están reglamentados desde el 2004), lo que constituye otra barrera más debido a las resistencias o desinformación que existen entre algunos profesionales de la salud (MORAN FAÚNDES, 2010; GALVÃO et al., 2000; MARÍN, 2004; ROMERO MEZARINA et al., 2007).

Dado el escaso período de tiempo que ha transcurrido desde la aprobación de la ley, la información estadística con la que se cuenta ha sido escasa. Incluso desde la introducción de los primeros anticonceptivos de emergencia en el país, nunca se publicaron datos oficiales relacionados con su disponibilidad y acceso en los servicios públicos de salud. Esto produjo 
una carencia de información sobre el nivel de demanda de la población usuaria, así como de las características sociodemográficas de la misma, elemento esencial para la construcción y el diseño de políticas públicas bajo el enfoque de los derechos sexuales y reproductivos.

A raíz de esta situación, una de las principales demandas de los movimientos feministas y de mujeres en Chile ha sido acceder a la información oficial sobre la situación de la anticoncepción de emergencia en los servicios de salud, a fin de monitorear su disponibilidad y accesibilidad.

Ante esta demanda, el Departamento de Estadísticas e Información de Salud, dependiente del Ministerio de Salud de Chile, recientemente ha publicado los primeros datos que se conocen respecto de la consulta y entrega de anticoncepción de emergencia en el país. Esta información resulta crucial para comprender los procesos sociales, políticos y económicos que estructuran el acceso a la anticoncepción de emergencia en Chile, en especial desde un marco que reconoce el vínculo entre las desigualdades socioeconómicas y la estructuración del género, la sexualidad y la reproducción.

De este modo, por primera vez desde que la anticoncepción de emergencia ingresó al país hace ya más de diez años, se cuenta con información cuantitativa susceptible de ser cruzada con datos sociodemográficos a fin de caracterizar la población demandante de este método y monitorear los niveles de consultas en el sistema de salud de Chile. ${ }^{1}$

En consecuencia, el presente trabajo tiene como finalidad analizar los datos disponibles de consulta a la anticoncepción de emergencia en el sistema nacional de servicios de salud. Para esto, se evalúa la incidencia de los factores socioeconómicos sobre los niveles de consulta de la población respecto de este método anticonceptivo, considerando especialmente el nivel de ingresos, el nivel educacional y la zona geográfica de residencia.

\section{La estructuración de la fecundidad y el uso de anticoncepción}

Las tasas globales de fecundidad (TGF) han experimentado un franco descenso en América Latina desde la década de los setentas. El caso de Chile no ha sido la excepción, y las proyecciones de población muestran que la fecundidad ha tendido a bajar en las últimas décadas (DONOSO; CARVAJAL; DOMÍNGUEZ, 2009), pasando de una TGF de 2,8 en el quinquenio 1975-1980 a una de 1,9 en el de 2005-2010, según las estimaciones del Centro Latinoamericano y Caribeño de Demografía de la CEPAL. Esto condice con la etapa avanzada de la primera transición demográfica en la que se encuentra actualmente el país (SCHKOLNIK, 2004; BERTRANOU, 2008), según la cual las tasas de fecundidad y mortalidad experimentan aún una sostenida disminución.

Sin embargo, esta tendencia no ha sido igual en todos los grupos etarios. En el caso del grupo conformado por adolescentes de entre 15 y 19 años de edad, si bien su tasa

\footnotetext{
1 Esta investigación utilizó información de la Encuesta de Caracterización Socioeconómica Nacional. El autor agradece al Ministerio de Planificación, propietario intelectual de la Encuesta, haberle permitido disponer de la base de datos. Todos los resultados del estudio son de responsabilidad del autor y en nada comprometen a dicho Ministerio.
} 
específica de fecundidad había mantenido una baja relativamente constante a lo largo de las últimas décadas, a partir del año 2004 la situación se revirtió, comenzando a experimentar un aumento que se ha sostenido en los años siguientes. En el caso del grupo etario de 20 a 44 años, este aumento recién se observó a partir del año 2007, tal como se observa en el Gráfico 1.

\section{GRÁFICO 1}

Tasas de fecundidad (1), según grupos de edad de la madre

Chile - 1999-2008

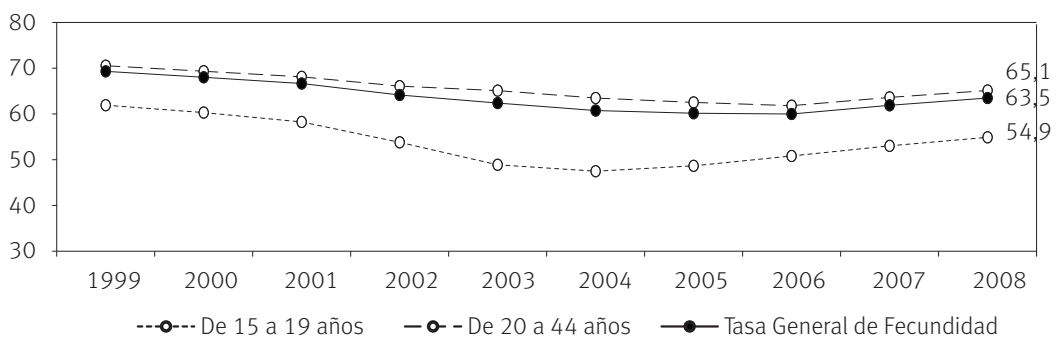

Fuente: Instituto Nacional de Estadísticas; Departamento de Estadísticas e Información de Salud del Ministerio de Salud (http://deis.minsal. $\mathrm{cl} /$ index.asp).

(1) Por mil nascidos vivos

Los comportamientos reproductivos tienden además a estructurarse en función de otras variables específicas, algunas asociadas a factores contextuales. Así, diversos estudios han mostrado la existencia de una relación estructurante entre las características socioeconómicas y la reproducción. En una investigación realizada por Di Cesare, se encontró que el estatus económico, el nivel educacional y el área de residencia (rural o urbana) tienen una incidencia directa sobre las conductas reproductivas de las poblaciones latinoamericanas, donde la pobreza, el bajo nivel educativo y la ruralidad inciden incrementando la probabilidad de tener hijos en las mujeres (DI CESARE, 2007). Similares resultados observó Schkolnik (2004) en siete países latinoamericanos, resaltando la especial centralidad de la educación de la madre en el diferencial de fecundidad. Para el caso de Chile, estas desigualdades también existen, aunque los datos censales han mostrado una tendencia hacia la convergencia de las tasas generales de fecundidad entre los distintos grupos socioeconómicos (RODRíGUEZ, 2005).

Sin embargo, entre las adolescentes menores de 20 años de edad los patrones de distribución desigual de la fecundidad tienden a acentuarse. Rodríguez, utilizando microdatos de los censos de 1992 y 2002 en Chile, mostró que las tasas de fecundidad en mujeres de entre 15 y 19 años de edad son mayores entre aquellas que viven en zonas rurales y que pertenecen al tercil socioeconómico más bajo, tendencia que no ha podido ser revertida a lo largo del tiempo (RODRÍGUEZ, 2005). Otro estudio demostró que las adolescentes de entre 10 y 14 años de edad que residen en comunas que reciben una baja proporción de raciones alimenticias, elemento asociado a un alto nivel socioeconómico de la población, tienen menor probabilidad de ser madres que aquellas que residen en 
comunas con mayores proporciones de raciones alimenticias (MOLINA; MOLINA; GONZÁLEZ, 2007). Este fenómeno es similar al observado en otras partes del partes del mundo, donde se ha corroborado la relación entre pobreza, educación y fecundidad para las poblaciones adolescentes (IMAMURA et al., 2007).

Así como se ha destacado la importancia de los factores contextuales que estructuran la fecundidad en función de las desigualdades socioeconómicas, geográficas y educacionales, la literatura ha dado una especial relevancia a los denominados “determinantes próximos”, es decir, aquellos que se estima que definen de manera directa la probabilidad de tener hijos. Si bien han sido diversos los determinantes próximos detectados por las investigaciones sociodemográficas, tales como los índices de infertilidad o la iniciación sexual, en general los patrones de uso de métodos anticonceptivos y los índices de aborto espontáneo o inducido (factor que en Chile es difícil de calcular dado el carácter ilegal del aborto) han sido destacados como los determinantes de mayor impacto sobre la fecundidad, y en especial sobre la población adolescente (BONGAARTS; MAULDIN, PHILLIPS, 1990; BAY; DEL POPOLO; FERRANDO, 2003; RODRÍGUEZ, 2005; RODRIGUEZ; DI CESARE, 2010).

Chile no cuenta con una fuente de información única y confiable para analizar estas variables. Sin embargo, la Encuesta Nacional de Juventud entrega ciertas luces respecto del acceso y uso de métodos anticonceptivos por parte de la población de entre 15 y 29 años en Chile. Al respecto, aunque se ha evidenciado un incremento en el uso de anticonceptivos entre los y las jóvenes iniciados sexualmente (DIDES; BENAVENTE; MORÁN, 2009), su uso varía significativamente en función del estrato socioeconómico y la zona geográfica de residencia (urbana/rural). Así, mientras el 86,4\% de los y las jóvenes del sector socioeconómico alto declaran haber utilizado un método anticonceptivo en su última relación sexual, esta cifra desciende al 64,9\% entre aquellos pertenecientes a los sectores más bajos. Esta tendencia se repite en lo que respecta al uso de un método de prevención durante la iniciación sexual (INJUV, 2010). Del mismo modo, la Encuesta Calidad de Vida y Salud del año 2006 evidenció que mientras el 81,9\% de las personas de más bajos recursos indica no utilizar preservativo durante sus relaciones sexuales, esta cifra desciende al 69,1\% en el caso de las personas de más alto nivel socioeconómico (AGUILERA et al., 2006). Pareciera ser así que el acceso y uso de métodos de prevención del embarazo queda supeditado a las desigualdades y exclusiones propias de la estructura socioeconómica del país, en especial entre los sectores adolescentes (RODRIGUEZ; DI CESARE, 2010). Esto especialmente considerando que en Chile se ha demostrado que la gran mayoría de las personas, y en particular las jóvenes, tienden a adquirir anticonceptivos mediante su compra en farmacias (70\%), lo que supone un acceso diferenciado en base al poder adquisitivo de cada cual (INJUV, 2010).

En esta línea, las políticas públicas integrales de información, educación y aseguramiento del acceso a métodos anticonceptivos modernos y eficaces, incluyendo la anticoncepción de emergencia, cobran una especial relevancia en la prevención del 
embarazo no deseado y en garantizar el derecho a las personas sobre su propio cuerpo, en especial en contextos de alta desigualdad como es el caso de los países latinoamericanos (DIDES; BENAVENTE; MORÁN, 2009; RODRIGUEZ; DI CESARE, 2010; BAY; DEL POPOLO; FERRANDO, 2003; RAYMOND; TRUSSELL; POLIS, 2007; FAÚNDES et al., 2007). Estas políticas se vuelven cruciales en la superación de las inequidades sociales, en la medida que permiten a los sectores tradicionalmente excluidos una mayor accesibilidad a las tecnologías de prevención y una mayor información respecto de su uso.

Pero el enfoque en torno a las políticas públicas no debe quedarse estancado únicamente en la calidad y efectivización de la oferta (RODRIGUEZ; DI CESARE, 2010). Se hace necesario conocer también a la población demandante y la heterogeneidad que subyace a ésta, a fin de garantizar un real acceso a la anticoncepción y promover políticas eficaces de prevención del embarazo no deseado para los distintos subgrupos poblacionales. Esto supone la necesidad de contar con información de calidad respecto a las características de los sectores demandantes de anticoncepción de emergencia, lo que permitiría conocer el efectivo nivel de acceso, el perfil de demanda y los condicionantes estructurales que constituyen la misma.

\section{Metodología}

Recientemente, y por primera vez desde que la anticoncepción de emergencia fue introducida en el país, el Ministerio de Salud ha construido una base de datos que consigna las consultas y las entregas de este método en el Sistema Nacional de Servicios de Salud. La información disponible abarca el período de enero de 2010 hasta diciembre del mismo año, lo que además constituye la primera fuente de datos oficial desde que entró en vigencia la ley 20.418 en el país.

El Sistema Nacional de Servicios de Salud está conformado por aquellos servicios dependientes del Ministerio de Salud, por lo que se excluyen datos de los prestadores privados de salud por no existir información unificada sobre consultas por anticoncepción de emergencia en dicho sector.

Para analizar la demanda por anticoncepción de emergencia se utilizó la información disponible de consultas de este método consignada a nivel comunal. ${ }^{2}$ Esto permitió caracterizar a la población demandante de manera indirecta, esto es, cruzando la información con otros datos comunales de interés. En este sentido, la unidad de análisis fueron las comunas donde se registraron consultas por anticoncepción de emergencia durante el 2010, lo que constituyó un total de 247 comunas. Se excluyeron las comunas que no registraron consultas, debido a que se desconocen los motivos por los que no existe dicho registro, los cuales pueden ir desde una efectiva falta de solicitudes de anticoncepción hasta problemas

\footnotetext{
$\overline{2}$ Chile es un país con un régimen político-administrativo de tipo unitario, dividido en 15 regiones. Cada región está conformada por un conjunto de comunas o municipalidades, lideradas por un alcalde o alcaldesa que es elegido cada cuatro años mediante la realización de elecciones populares. En total, el país se divide en 345 comunas.
} 
de consignación de datos o actualización de la información oficial. La base de datos final utilizada en los análisis del presente estudio consignó la demanda de anticoncepción de emergencia para más del $85 \%$ de la población total de mujeres en edad fértil de entre 15 y 44 años de edad en Chile.

A fin de analizar la demanda de cada comuna se calculó una tasa de consultas por anticoncepción de emergencia a nivel comunal. Para esto, se estableció el cociente entre el total de consultas comunales por anticoncepción de emergencia en el año 2010 y la población comunal estimada de mujeres en edad fértil (entre 15 y 44 años) para el mismo año, por mil. Los datos de la población comunal provinieron de las estimaciones poblacionales del Instituto Nacional de Estadísticas de Chile (www.ine.cl). Se establecieron además tasas según la edad de la solicitante.

Siguiendo a Di Cesare, la medición de las características socioeconómicas de la población como factor estructurante de los comportamientos reproductivos consideró tres variables centrales, a saber: el estatus económico, el nivel de educación y el lugar de residencia (rural/ urbana) (DI CESARE, 2007).

El estatus económico de la población comunal fue caracterizado utilizando los datos de la Encuesta CASEN de Caracterización Socioeconómica Nacional (MIDEPLAN, 2009) del año 2009. A fin de establecer la cantidad de personas que poseen un bajo estatus económico, se consignó el porcentaje de la población de cada comuna cuyo ingreso autónomo per cápita se sitúa en el primer quintil de los ingresos regionales. Así, al considerar los ingresos regionales, se controlan automáticamente las variaciones que pueden darse entre cada una de las regiones del país.

Para establecer el nivel educacional de la población de cada comuna se utilizaron los datos de la misma encuesta, consignando el porcentaje de personas de 20 años o más sin educación secundaria completa, siguiendo una metodología similar a la utilizada por Pantelides y Binstock (2007).

Finalmente, la operacionalización de la zona de residencia se estableció utilizando las proyecciones de población rural del Instituto Nacional de Estadísticas de Chile. Los asentamientos rurales se definieron como aquellos que se encuentran concentrados o dispersos con un total de mil o menos habitantes, o entre 1.001 y 2.000 habitantes en los que menos del $50 \%$ de la población económicamente activa se dedica a actividades secundarias (GONZÁLEZ; ESPINA, 2001). Siguiendo esto, se calculó el porcentaje de población rural estimada a nivel comunal para el año 2010.

La información de cada una de estas tres últimas variables (estatus económico, nivel educacional y zona de residencia) fue dividida en quintiles, para luego calcular la tasa promedio de consultas por anticoncepción de emergencia en cada uno de éstos. De este modo, el primer quintil de cada variable representa aquellas comunas con una escasa proporción de población comunal con bajos ingresos, bajo porcentaje de habitantes sin secundario completo o poca población rural, mientras que el último quintil representa las comunas con peores indicadores socioeconómicos. 


\section{Resultados}

Tal como muestra la Tabla 1, durante el 2010 se entregaron un total de 4.374 dosis de anticoncepción de emergencia en los establecimientos del Sistema Nacional de Servicios de Salud, según los datos registrados por el Departamento de Estadísticas e Información de Salud del Ministerio de Salud.

TABLA 1

Consultas por anticoncepción de emergencia (AE) en mujeres en edad fértil, según características seleccionadas

Chile -2010

\begin{tabular}{|c|c|c|c|}
\hline Características seleccionadas & N. & $\%$ & $\begin{array}{c}\text { Tasa media (por } \\
\text { mil mujeres) }\end{array}$ \\
\hline Total de consultas por AE & 4.374 & 100,00 & - \\
\hline \multicolumn{4}{|l|}{ Situación de entrega de AE } \\
\hline Consultas con entrega de AE & 3.218 & 73,57 & - \\
\hline Consultas sin entrega de $\mathrm{AE}$ & 1.156 & 26,43 & - \\
\hline \multicolumn{4}{|c|}{ Personal de salud que atendió a la solicitante de AE } \\
\hline Consultas atendidas por médico/a & 486 & 11,11 & - \\
\hline Consultas atendidas por matrona & 3.888 & 88,89 & - \\
\hline \multicolumn{4}{|l|}{ Consultas de AE en mujeres por grupos de edad } \\
\hline 15 a 19 años & 1.489 & 34,04 & 4,27 \\
\hline 20 a 44 años & 2.885 & 65,96 & 2,10 \\
\hline 15 a 44 años & 4.374 & 100,00 & 2,53 \\
\hline
\end{tabular}

Fuente: Ministerio de Salud; Instituto Nacional de Estadísticas de Chile; Encuesta CASEN de Caracterización Socioeconómica Nacional (MIDEPLAN, 2009).

En el 73,57\% del total de las consultas se entregó anticoncepción de emergencia a la solicitante, mientras que en casi una cuarta parte esto no ocurrió. Los motivos de no entrega se desconocen.

Asimismo, el 88,89\% de las consultas fueron atendidas por matronas, mientras que sólo un $11,11 \%$ por médicos o médicas, lo que muestra el rol central que juegan las matronas en los servicios de acceso a la anticoncepción en el país.

La tasa media de consultas por anticoncepción de emergencia en mujeres en edad fértil (15 a 44 años) fue de 2,53 consultas por cada mil mujeres. Sin embargo, se observan importantes diferencias entre los distintos tramos etarios, ya que esta cifra llega a 4,27 en mujeres adolescentes de entre 15 y 19 años, y sólo a 2,1 en mujeres de 20 a 44. Asimismo, más de la tercera parte de las consultas del 2010 fueron realizadas por mujeres menores de 20 años.

Los datos regionales muestran que la mayor tasa de consultas por anticoncepción de emergencia se sitúa en las regiones de Los Lagos, Aisén, Arica Parinacota y Magallanes, lo que supone una mayor tasa en las zonas geográficas extremas del país. Por otra parte, las menores tasas de consultas se evidencian en las regiones de Antofagasta, de Coquimbo y en la Región Metropolitana de Santiago. 


\section{GRÁFICO 2}

Tasas medias de consultas por anticoncepción de emergencia en mujeres en edad fértil (1), por regiones

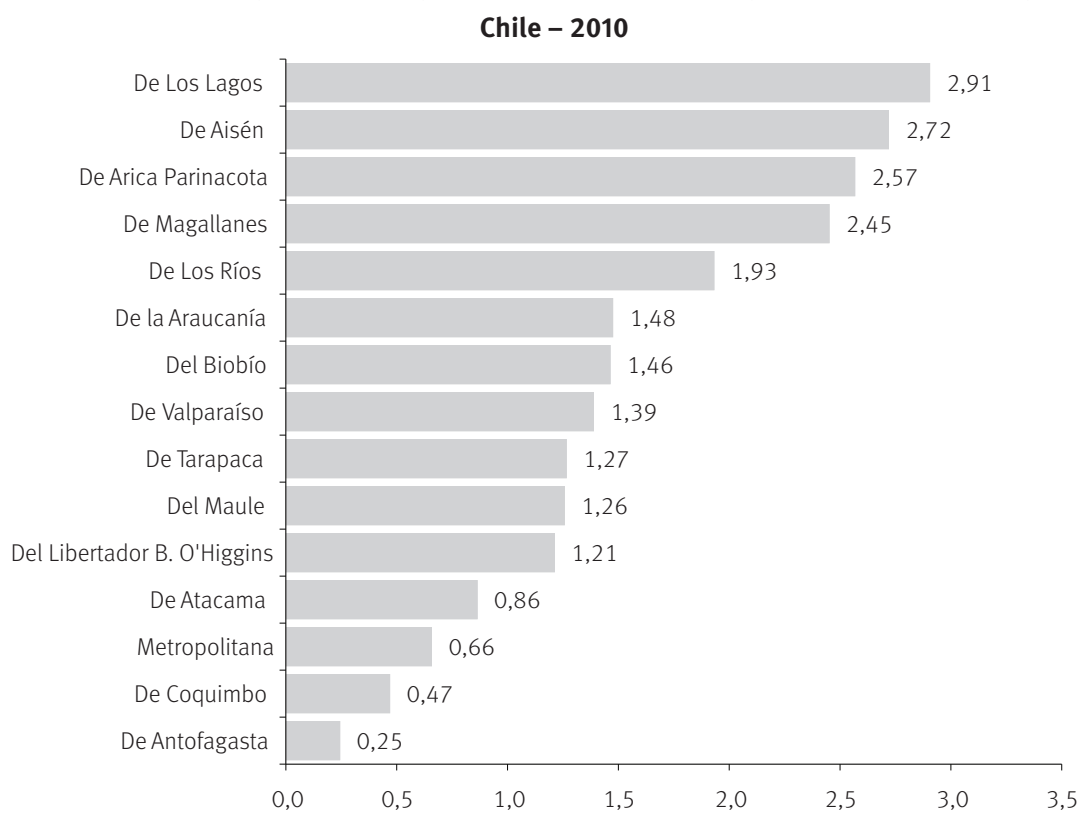

Fuente Ministerio de Salud; Instituto Nacional de Estadísticas de Chile; Encuesta CASEN de Caracterización Socioeconómica Nacional (MIDEPLAN, 2009).

(1) Por mil mujeres.

TABLA 2

Tasas medias de consultas por anticoncepción de emergencia en mujeres en edad fértil, por grupos de edad, según quintiles de población comunal con estatus económico bajo

Chile - 2010

\begin{tabular}{|c|c|c|c|c|c|c|}
\hline \multirow{2}{*}{$\begin{array}{l}\text { Quintiles de las } \\
\text { comunas según } \\
\text { proporción de } \\
\text { población comunal } \\
\text { con estatus } \\
\text { económico bajo }\end{array}$} & \multirow{2}{*}{$\begin{array}{c}\text { Valor } \\
\text { percentil }\end{array}$} & \multirow{2}{*}{ N. } & \multirow{2}{*}{$\begin{array}{l}\text { Porcentaje } \\
\text { promedio de } \\
\text { población } \\
\text { comunal } \\
\text { con estatus } \\
\text { económico bajo }\end{array}$} & \multicolumn{3}{|c|}{$\begin{array}{l}\text { Tasa media de consultas por AE } \\
\text { (por mil mujeres) }\end{array}$} \\
\hline & & & & 15 a 19 años & 20 a 44 años & 15 a 44 años \\
\hline Quintil 1 & 17,78 & 49 & 13,74 & 3,45 & 1,50 & 1,87 \\
\hline Quintil 2 & 21,83 & 50 & 20,03 & 4,53 & 1,64 & 2,17 \\
\hline Quintil 3 & 25,71 & 49 & 23,91 & 4,50 & 1,68 & 2,26 \\
\hline Quintil 4 & 30,79 & 50 & 28,31 & 4,84 & 2,00 & 2,56 \\
\hline Quintil 5 & 58,19 & 49 & 36,75 & 4,02 & 2,20 & 2,54 \\
\hline Total & - & 247 & 24,55 & 4,27 & 1,80 & 2,28 \\
\hline
\end{tabular}

Fuente: Ministerio de Salud; Instituto Nacional de Estadísticas de Chile; Encuesta CASEN de Caracterización Socioeconómica Nacional (MIDEPLAN, 2009).

Al observar los datos de la proporción de población comunal con un estatus económico bajo, cruzados con las tasas de consultas por anticoncepción de emergencia a nivel comunal, se observa en general que a medida que aumenta la proporción de población con bajos 
ingresos aumenta también la tasa de consultas, con un leve descenso en el último quintil respecto del cuarto.

\section{GRÁFICO 3}

Tasas medias de consultas por anticoncepción de emergencia en mujeres en edad fértil (1), por grupos de edad, según quintiles de población comunal con estatus económico bajo

Chile - 2010

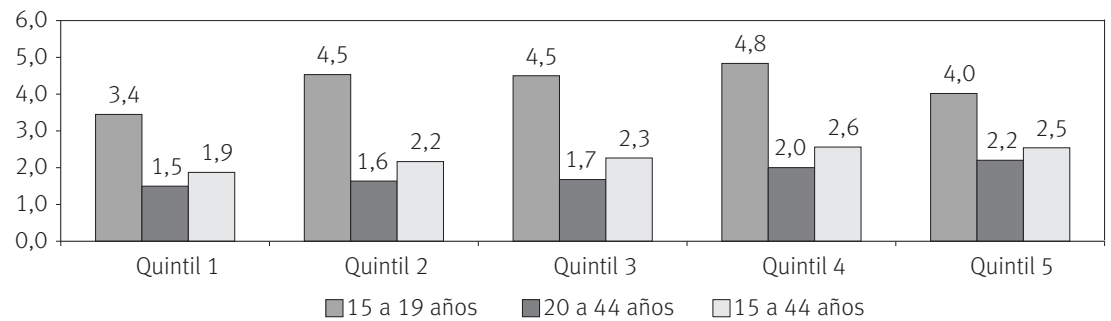

Fuente: Ministerio de Salud; Instituto Nacional de Estadísticas de Chile; Encuesta CASEN de Caracterización Socioeconómica Nacional (MIDEPLAN, 2009)

(1) Por mil mujeres

Las comunas situadas en el primer quintil, esto es, con la menor proporción de población con un bajo estatus económico, poseen una tasa media de 1,87 consultas por anticoncepción de emergencia por cada mil mujeres en edad fértil, valor que aumenta 2,54 en el caso de las comunas situadas en el último quintil.

Para el grupo específico de mujeres de 20 a 44 años, el aumento de la tasa de consultas por anticoncepción de emergencia es sostenido a medida que aumenta la proporción de población comunal con estatus económico bajo.

Sin embargo, en el grupo de adolescentes de 15 a 19 años, la relación entre los niveles de ingresos y las consultas por anticoncepción de emergencia se rompe, ya que en este sector poblacional las mayores tasas de consultas se sitúan en el cuarto $(4,84)$ y el segundo $(4,53)$ quintil, mientras que los quintiles de los extremos presentan las tasas más bajas.

TABLA 3

Tasas medias de consultas por anticoncepción de emergencia en mujeres en edad fértil, por grupos de edad, según quintiles de población rural a nivel comunal

Chile - 2010

\begin{tabular}{|c|c|c|c|c|c|c|}
\hline \multirow{2}{*}{$\begin{array}{l}\text { Quintiles de } \\
\text { comunas según } \\
\text { proporción de } \\
\text { población rural }\end{array}$} & \multirow{2}{*}{$\begin{array}{c}\text { Valor } \\
\text { percentil }\end{array}$} & \multirow{2}{*}{ N. } & \multirow{2}{*}{$\begin{array}{l}\text { Porcentaje } \\
\text { promedio de } \\
\text { población rural } \\
\text { comunal }\end{array}$} & \multicolumn{3}{|c|}{$\begin{array}{c}\text { Tasa media de consultas por AE (por mil } \\
\text { mujeres) }\end{array}$} \\
\hline & & & & 15 a 19 años & 20 a 44 años & 15 a 44 años \\
\hline Quintil 1 & 4,52 & 49 & 0,64 & 1,97 & 0,95 & 1,13 \\
\hline Quintil 2 & 22,60 & 49 & 11,78 & 3,74 & 1,88 & 2,24 \\
\hline Quintil 3 & 40,61 & 50 & 31,36 & 4,57 & 1,84 & 2,35 \\
\hline Quintil 4 & 56,95 & 50 & 48,33 & 5,17 & 2,03 & 2,64 \\
\hline Quintil 5 & 100,00 & 49 & 71,71 & 5,88 & 2,31 & 3,03 \\
\hline Total & - & 247 & 32,82 & 4,27 & 1,80 & 2,28 \\
\hline
\end{tabular}

Fuente: Ministerio de Salud; Instituto Nacional de Estadísticas de Chile; Encuesta CASEN de Caracterización Socioeconómica Nacional (MIDEPLAN, 2009) 


\section{GRÁFICO 4}

Tasas medias de consultas por anticoncepción de emergencia en mujeres en edad fértil (1), por grupos de edad, según quintiles de población rural a nivel comunal

Chile - 2010

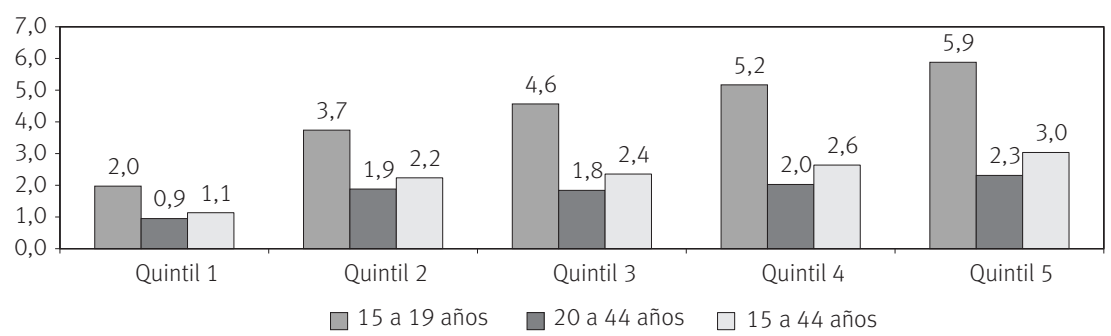

Fuente: Ministerio de Salud; Instituto Nacional de Estadísticas de Chile; Encuesta CASEN de Caracterización Socioeconómica Nacional (MIDEPLAN, 2009).

(1) Por mil mujeres.

Los datos de comunas según su población rural, agrupados en quintiles, muestran que a mayor proporción de ruralidad la tasa de consultas por anticoncepción de emergencia tiende a aumentar. En este sentido, en el primer quintil la tasa de consultas en mujeres de 15 a 44 años llega a ser de 1,13 por cada mil mujeres en edad fértil, cifra que aumenta casi tres veces en el último quintil, llegando a 3,03. Esta relación se mantiene tanto en el grupo de adolescentes como en las mujeres adultas en edad fértil, donde se observa un aumento sostenido de la tasa de consultas a medida que aumenta la población rural comunal.

TABLA 4

Tasas medias de consultas por anticoncepción de emergencia en mujeres en edad fértil, por grupos de edad, según quintiles de población comunal de 20 años o más con educación secundaria incompleta a nivel comunal

Chile - 2010

\begin{tabular}{|c|c|c|c|c|c|c|}
\hline \multirow{2}{*}{$\begin{array}{l}\text { Quintiles de } \\
\text { comunas según } \\
\text { población de } \\
20 \text { años o más } \\
\text { con educación } \\
\text { secundaria } \\
\text { incompleta }\end{array}$} & \multirow{2}{*}{$\begin{array}{l}\text { Valor } \\
\text { percentil }\end{array}$} & \multirow{2}{*}{ N. } & \multirow{2}{*}{$\begin{array}{c}\text { Porcentaje } \\
\text { promedio de } \\
\text { población comunal } \\
\text { de } 20 \text { años o más } \\
\text { con secundario } \\
\text { incompleto }\end{array}$} & \multicolumn{3}{|c|}{$\begin{array}{c}\text { Tasa media de consultas por AE (por mil } \\
\text { mujeres) }\end{array}$} \\
\hline & & & & 15 a 19 años & 20 a 44 años & 15 a 44 años \\
\hline Quintil 1 & 46,66 & 49 & 37,19 & 2,53 & 1,23 & 1,47 \\
\hline Quintil 2 & 57,10 & 50 & 51,69 & 3,07 & 1,64 & 1,91 \\
\hline Quintil 3 & 64,70 & 49 & 60,83 & 4,93 & 1,76 & 2,37 \\
\hline Quintil 4 & 71,37 & 50 & 67,92 & 4,98 & 1,91 & 2,51 \\
\hline Quintil 5 & 87,57 & 49 & 75,58 & 5,85 & 2,48 & 3,15 \\
\hline Total & - & 247 & 58,65 & 4,27 & 1,80 & 2,28 \\
\hline
\end{tabular}

Fuente: Ministerio de Salud; Instituto Nacional de Estadísticas de Chile; Encuesta CASEN de Caracterización Socioeconómica Nacional (MIDEPLAN, 2009).

En cuanto a la relación entre educación y consultas por anticoncepción de emergencia, a medida que aumenta la proporción comunal de personas de 20 años o más con educación secundaria incompleta, aumenta la tasa de consultas en mujeres en edad fértil. Esta relación 
se vuelve más fuerte en el grupo de adolescentes de 15 a 19 años, ya que la tasa de consultas en el primer quintil de comunas llega a 2,53, aumentando a 5,85 en el último quintil.

\section{GRÁFICO 5}

Tasas medias de consultas por anticoncepción de emergencia en mujeres en edad fértil (1), por grupos de edad, según quintiles de población comunal de 20 años o más con educación secundaria incompleta a nivel comunal

Chile - 2010

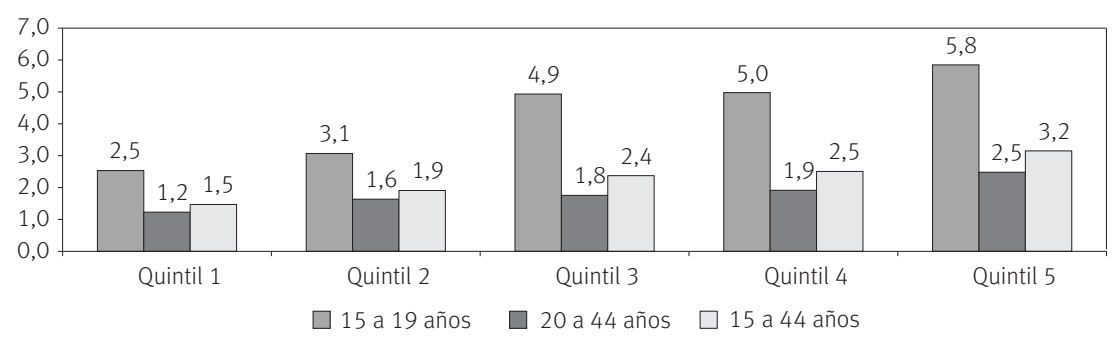

Fuente: Ministerio de Salud; Instituto Nacional de Estadísticas de Chile; Encuesta CASEN de Caracterización Socioeconómica Nacional (MIDEPLAN, 2009).

(1) Por mil mujeres.

\section{GRÁFICO 6}

Relaciones entre el nivel socioeconómico, la ruralidad y la escolaridad a nivel comunal Chile - 2010
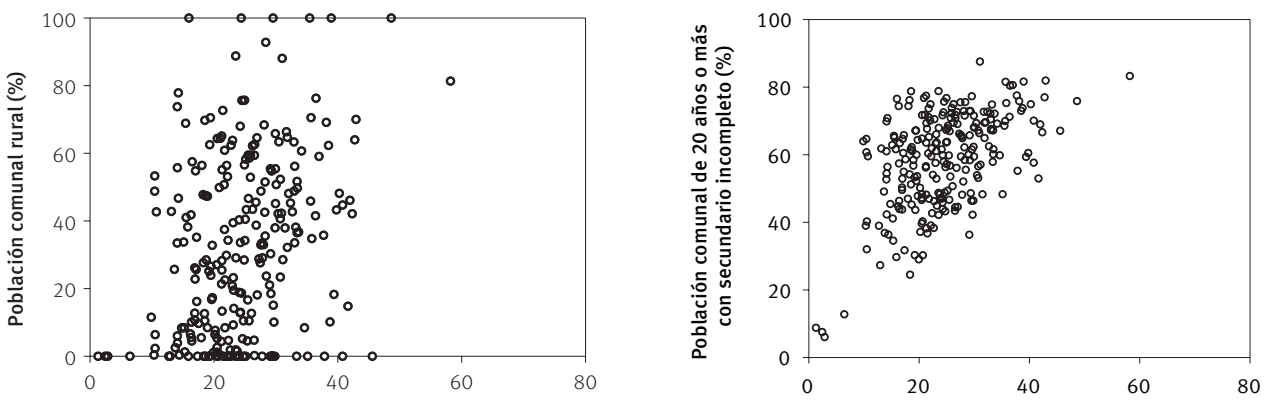

Población comunal con nivel socioeconómico bajo (\%)

Población comunal con nivel socioeconómico bajo (\%)

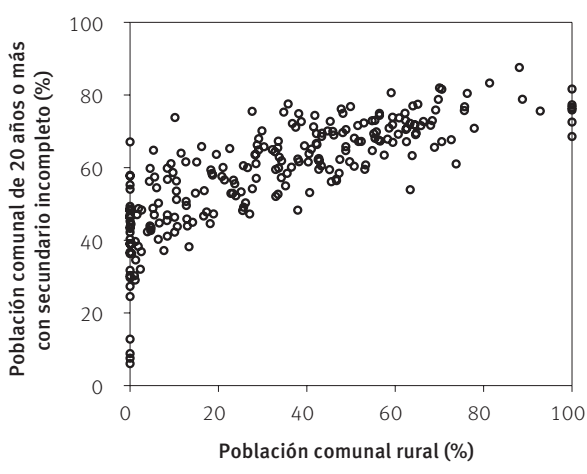

\begin{tabular}{|l|c|c|c|}
\hline \multicolumn{4}{|c|}{ Coeficientes de correlación de Pearson } \\
\hline & $\begin{array}{c}\text { Población } \\
\text { comunal con nivel } \\
\text { socioeconómico } \\
\text { bajo }\end{array}$ & $\begin{array}{c}\text { Población } \\
\text { comuna } \\
\text { rural }\end{array}$ & $\begin{array}{c}\text { Población } \\
\text { comunal de } \\
20 \text { años o más } \\
\text { con secundario } \\
\text { incompleto }\end{array}$ \\
\hline $\begin{array}{l}\text { Población } \\
\text { comunal con nivel } \\
\text { socioeconómico } \\
\text { bajo }\end{array}$ & 1,00 & 0,32 & 0,52 \\
\hline $\begin{array}{l}\text { Población comuna } \\
\text { rural }\end{array}$ & 0,32 & 1,00 & 0,78 \\
\hline $\begin{array}{l}\text { Población comunal } \\
\text { de 20 años o más } \\
\text { con secundario } \\
\text { incompleto }\end{array}$ & 0,52 & 0,78 & 1,00 \\
\hline
\end{tabular}

Fuente: Ministerio de Salud; Instituto Nacional de Estadísticas de Chile; Encuesta CASEN de Caracterización Socioeconómica Nacional (MIDEPLAN, 2009). 
Tal como se desprende de los gráficos de dispersión y los coeficientes de correlación de Pearson, las tres variables analizadas como factores determinantes de las consultas por anticoncepción de emergencia tienen relaciones lineales positivas entre sí. La relación más débil se da entre el nivel socioeconómico con la ruralidad, donde el coeficiente de correlación llega a 0,32, mientras que el nivel de escolaridad presenta fuertes relaciones con las otras dos variables, con un coeficiente de 0,78. Esto implica la pertinencia de considerar las tres variables como indicadores de una misma dimensión, esto es, el nivel socioeconómico de la población comunal, y no como factores independientes entre sí.

\section{Discusión y reflexiones finales}

La literatura ha mostrado la existencia de importantes relaciones entre los comportamientos reproductivos y determinados factores contextuales de orden económico y social. En América Latina se ha puesto de relieve la relación entre la fecundidad de las mujeres con el estatus económico, el nivel educacional y el lugar de residencia, destacándose la pobreza, la escolaridad incompleta y la ruralidad como variables que tienden a aumentar las probabilidades de ser madre (DI CESARE, 2007). Esto se observa especialmente en el caso de las adolescentes, donde las estructuras socioeconómicas tienden a determinar fuertemente los patrones de reproducción de las menores de 20 años (IMAMURA et al., 2007; RODRÍGUEZ, 2005; RODRÍGUEZ; DI CESARE, 2010).

La estructuración de los comportamientos reproductivos en función de factores socioeconómicos supone una desigual distribución y acceso a métodos de prevención del embarazo, elemento que ha sido destacado por la literatura como uno de los más importantes determinantes próximos de la fecundidad (BAY; DEL POPOLO; FERRANDO, 2003; RODRÍGUEZ, 2005; SCHKOLNIK, 2004). Esto supone la necesidad de orientar los esfuerzos en lograr mejorar los niveles de acceso a métodos anticonceptivos y servicios de aborto seguro a la totalidad de la población, superando las desigualdades estructurales que median dicha accesibilidad, situación que se expresa con especial fuerza en los contextos latinoamericanos.

En el caso específico de la entrega de anticoncepción de emergencia, los diversos estudios regionales han destacado la existencia de barreras en el acceso desde distintas perspectivas (FAÚNDES et al., 2007; SOUZA; BRANDÃO, 2009). Un estudio realizado el 2006 en la ciudad de São Pablo mostraba importantes limitaciones en la distribución de anticoncepción de emergencia en las unidades básicas de salud (FIGUEIREDO et al., 2007), situación similar a la observada en Chile durante el 2009, donde sólo el 50,5\% de las comunas que contaban con servicios de salud municipalizada distribuían el método (DIDES; BENAVENTE; MORÁN, 2010).

Asimismo, otros estudios han destacado un alto nivel de desconocimiento por parte del personal de salud respecto de la condición legal de la anticoncepción de emergencia en sus países o de los mecanismos de acción de ésta, subrayando incluso la presencia de resistencias fundadas sobre creencias religiosas (GALVÃO et al., 2000; MARÍN, 2004; ROMERO MEZARINA et al., 2007). Esto hace que el acceso a este método de prevención del embarazo 
se vea en riesgo, lo que sin dudas tiende a afectar con mayor intensidad a las poblaciones más vulneradas y excluidas de los sistemas de protección social.

En lo que respecta a la demanda y uso, según los datos de la VI Encuesta Nacional de Juventud, el 7,3\% de las mujeres en Chile de entre 15 y 29 años declaró haber utilizado la anticoncepción de emergencia en los últimos 12 meses. La información desagregada por edades mostró un uso diferenciado según tramos etarios, ya que mientras el 10,4\% de las adolescentes de entre 15 y 19 años señaló haber utilizado este método, esta cifra llegó sólo a un 8,2\% en el grupo de 20 a 24 años, y a 4,5\% en el de 25 a 29 (INJUV, 2010).

Estos resultados condicen con los hallazgos del presente estudio, donde se observa una fuerte tasa de demanda por anticoncepción de emergencia entre las adolescentes (4,27 por cada mil mujeres de la misma edad), más del doble de la observada en las mujeres de 20 a 44 años.

Asimismo, la baja prevalencia observada de uso de anticonceptivos en la última relación sexual entre las personas adolescentes, en comparación con el uso observado en jóvenes de 20 a 29 años (INJUV, 2010), condice con la alta tasa de demanda por anticoncepción de emergencia entre las mujeres entre 15 y 19 años de edad en el sector público de salud analizada en el presente estudio.

Respecto de la relación entre el uso de la anticoncepción de emergencia y el nivel socioeconómico, la Encuesta Nacional de Juventud muestra también que el uso de este método se concentra en los estratos más altos, mientras que en los sectores más bajos su uso desciende (INJUV, 2010). Esta tendencia ha sido observada también en otros lugares de la región latinoamericana. Un estudio realizado en las escuelas públicas de Pernambuco, en Brasil, demostró por ejemplo que los mayores niveles de conocimiento de la anticoncepción de emergencia se situaban entre los estudiantes con condiciones económicas más favorecidas, lo que hace presumir un mayor uso por parte de estos sectores respecto de aquellos con menos recursos (ARAÚJO; COSTA, 2009).

Estos últimos datos difieren de los observados en los resultados de la presente investigación, por cuanto la mayor demanda en el servicio nacional de salud se concentra en los sectores con peores indicadores socioeconómicos. Esto puede deberse al uso diferenciado que hacen las personas en Chile del sistema de salud pública y privado. En este sentido, algunos estudios han demostrado que casi la totalidad de las personas situadas en el quintil de ingresos más bajos son usuarias del sistema público de salud, mientras que la mayoría de aquellas pertenecientes al quintil más favorecido utilizan el sistema privado (LARRAÑAGA, 1997). De este modo, es posible que la mayor demanda por anticoncepción de emergencia observada en las comunas con mayor proporción de personas en situación socioeconómica baja se deba a un mayor uso del sistema público por parte de la población comunal.

Llama la atención el comportamiento registrado en este estudio en el grupo de adolescentes de 15 a 19 años respecto de la variable vinculada con los ingresos autónomos, donde la demanda por anticoncepción de emergencia tiende a concentrarse en los quintiles medios, mientras que los extremos presentan las tasas de demanda más bajas. Esto resulta 
aún más llamativo considerando que el comportamiento del tramo etario de 20 a 44 años tiende a mostrar un aumento sostenido en la demanda a medida que aumenta la proporción comunal de personas con estatus económico bajo, diferenciándose así del comportamiento observado en el grupo de adolescentes. Si bien los datos de la presente investigación son insuficientes para establecer las causas de estas conductas diferenciadas, es necesario considerar que las otras dos variables (nivel educacional y zona de residencia) mostraron que la mayor demanda por parte de las adolescentes se sitúa en las comunas con bajos niveles de escolaridad completa y con altos niveles de ruralidad, lo que denota la incidencia de las variables socioeconómicas sobre la demanda por anticoncepción de emergencia.

Los resultados de este estudio entregan algunas luces respecto de la estructuración de la demanda por la denominada "píldora del día después" en Chile en las instituciones del sistema nacional de servicios de salud. La demanda diferenciada en base a condicionantes socioeconómicos es el reflejo de un comportamiento reproductivo segmentado según estructuras de desigualdad de ingresos, de oportunidades en educación y de desarrollo geográfico. Son las personas con niveles socioeconómicos más bajos las que se ven mayormente afectadas por estas desigualdades al tener un menor acceso a tecnologías de contracepción eficaces, lo que explica en cierta forma la concentración de la demanda por anticoncepción de emergencia en estos sectores. Las políticas públicas deben apuntar a mejorar el acceso a este método, en especial a los sectores de adolescentes y a los menos favorecidos, a fin de contribuir desde esta dimensión a romper el círculo de la pobreza y garantizar el derecho de las personas a disponer libremente sobre su propio cuerpo en materia reproductiva.

Para esto, se hace indispensable profundizar la información oficial disponible, consignando las razones específicas por las que las mujeres consultan por anticoncepción de emergencia en los servicios de salud, los motivos por los que en algunas consultas no se entrega el método (26,4\% según los datos de este estudio) y las causales que explicarían las grandes diferencias en las tasas de consultas a nivel regional al interior del país.

\section{Referencias}

AGUILERA, X.; GONZÁLEZ, C.; GUERRERO, A.; HOFFMEISTER, L.; CÁRDENAS, P.; BURGOS, A.; MEDINA, B.; VALLEBUONA, C. II Encuesta de Calidad de Vida y Salud: informe de resultados. Total nacional. Santiago: Ministerio de Salud del Gobierno de Chile, 2006. Disponible en: 〈http://epi.minsal.cl/epi/ $\mathrm{html} /$ sdesalud/calidaddevida2006/Informe\%20Final\%20Encuesta\%20de\%20Calidad\%20de\%20 Vida\%20y\%20Salud\%202006.pdf>. Acceso en: 10 abr. 2011.

ARAÚJO, M. S. P.; COSTA, L. O. B. F. Comportamento sexual e contracepção de emergência entre adolescentes de escolas públicas de Pernambuco, Brasil. Cadernos de Saúde Pública, v. 25, n. 3 , p. 551-562, 2009. Disponible en: 〈http://www.scielosp.org/pdf/csp/v25n3/10.pdf〉. Acceso en: 10 abr. 2011.

BAY, G.; DEL POPOLO, F.; FERRANDO, D. Determinantes próximos de la fecundidad. Una aplicación a países latinoamericanos. Serie Población y Desarrollo, n. 43. Santiago: CEPAL, 2003.

BONGAARTS, J.; MAULDIN, W. P.; PHILLIPS, J. F. The demographic impact of family planning programs, Studies in Family Planning, v. 21, n. 6, p. 299-310, noviembre/diciembre 1990. 
BERTRANOU, E. Tendencias demográficas y protección social en América Latina y El Caribe. Serie Población y Desarrollo, n. 82. Santiago: CEPAL, 2008.

BUSQUETS, M. Contracepción de emergencia: efecto postfertilización del levonorgestrel. Revista Chilena de Obstetricia y Ginecología, v. 68, n. 2, p. 163-180, 2003. Disponible en: 〈http://www.scielo.cl/scielo. php?script=sci_arttext\&pid=S0717-75262003000200012\&lng=es\&nrm=iso>. Acceso en: 4 abr. 2011.

CASAS, L. Mi cuerpo no es realmente mío. In: BERGALLO, P. (Ed.). Derecho y propiedad. Buenos Aires: Libraria, 2009.

COOK, R. J.; ERDMAN, J. N.; DICKENS, B. M. Respecting adolescents' confidentiality and reproductive and sexual choices. International Journal of Gynecology and Obstetrics, v. 98, n. 2, p. 182-187, 2007.

CROXATTO, H. Gamete transport. In: ADASHI, E. Y.; ROCK, J. A.; ROSENWAKS, Z. (Eds.). Reproductive endocrinology, surgery, and technology. New York: Lippincot-Raven, 1996, p. 386-402.

Mecanismo de acción del levonorgestrel en la anticoncepción de emergencia. Revista

Chilena de Obstetricia y Ginecología, v. 69, n. 2, p. 157-162, 2004. Disponible en: 〈http://www. scielo.cl/scielo.php?script=sci_arttext\&pid=S0717-75262004000200011\&lng=es\&nrm=iso . Acceso en: 4 abr. 2011.

DI CESARE, M. Patrones emergentes en la fecundidad y la salud reproductiva y sus vínculos con la pobreza en América Latina y El Caribe. Serie Población y Desarrollo, n. 72. Santiago: CEPAL, 2007.

DIDES, C. Voces en emergencia: El discurso conservador y la píldora del día después. Santiago: FLACSO Chile, 2006.

DIDES, C.; BENAVENTE, M. C.; MORÁN, J. M. Diagnóstico de la situación del embarazo en la adolescencia en Chile, 2008. Santiago: Ministerio de Salud del Gobierno de Chile, UNFPA, FLACSO Chile, 2009. Disponible en: 〈http://issuu.com/flacso.chile/docs/embarazo_adolescente〉. Acceso en: 2 abr. 2011.

. Entrega de la píldora anticonceptiva de emergencia en el sistema de salud municipal, Chile: Estado de Situación. Santiago: FLACSO Chile, UNFPA, 2010. Disponible en: 〈http://www.flacso. cl/home/images/publicaciones/entregapildoraemergencia.pdf> Acceso en: 4 abr. 2011.

DONOSO, E.; CARVAJAL, J.; DOMÍNGUEZ, M. A. Reducción de la fecundidad y envejecimiento de la población de mujeres chilenas en edad fértil: 1990-2004. Revista Médica de Chile, v. 137, n. 6, p. 766-773, 2009 .

DURAND, M.; SEPPALA, M.; CRAVIOTO, M. C.; KOISTINEN, H.; KOISTINEN, R.; GONZÁLEZ-MACEDO, J.; LARREA, F. Late follicular phase administration of levonorgestrel as an emergency contraceptive changes the secretory pattern of glycodelin in serum and endometrium during the luteal phase of the menstrual cycle. Contraception, v. 71, n. 6, p. 451-457, 2005.

FAÚNDES, A.; TÁVARA, L.; BRACHE, V.; ALVAREZ, F. Emergency contraception under attack in Latin America: Response of the medical establishment and civil society. Reproductive Health Matters, v. 15, n. 29, p. 130-138, 2007.

FIGUEIREDO, R.; BASTOS, S.; SOARES, M. A.; LENZTELLES, J.; MIRANDA, M. Distribuição da contracepção de emergência na atenção básica de São Paulo: caracterização de oferta em PSF e UBS dos municípios do estado. Boletim do Instituto de Saúde, n. 42, p. 38-44, 2007. Disponible en: 〈http://www.isaude.sp.gov. br/smartsitephp/media/isaude/file/bis/bis42-tecnologia_completo.pdf>. Acceso en: 11 abr. 2011.

GALVÃO, L.; DÍAZ, J.; DÍAZ, M.; OSIS, M. J.; CLARK, S.; ELLERTSON, C. Anticoncepción de emergencia: conocimiento, actitudes y prácticas de los gineco-obstetras del Brasil. International Family Planning Perspectives, p. 2-6, 2000. Disponible en: 〈http://www.guttmacher.org/pubs/journals/2600200S. pdf>. Acceso en: 9 abr. 2011. 
GONZÁLEZ, D.; ESPINA, R. Urbanización y evolución de la población urbana de América Latina. 1950 1990. Boletín Demográfico, v. 33, Edición Especial, CEPAL, 2001. Disponible en: 〈http://www.eclac. org/cgi-bin/getProd.asp?xml=/publicaciones/xml/4/7394/P7394.xml\&xsl=/celade/tpl/p9f.xsl\&base=/ celade/tpl/top-bottom_minterna.xslt> Acceso en: 11 abr. 2011.

IMAMURA, M.; TUCKER, J.; HANNAFORD, P.; OLIVEIRA DA SILVA, M.; ASTIN, M.; WYNESS, L.; BLOEMENKAMP, K. W. M.; JAHN, A.; KARRO, H.; TEMMERMAN, M. Factors associated with teenage pregnancy in the European Union countries: a systematic review. European Journal of Public Health, v. 17, n. 6, p. 630-636, 2007. Disponible en: 〈http://eurpub.oxfordjournals.org/content/17/6/630.full.pdf+html?sid=cc97f2e6-843043ce-916a-c266480424c1〉. Acceso en: 7 abr. 2011.

INJUV. 6a Encuesta Nacional de Juventud. Santiago: INJUV, Gobierno de Chile, 2010. Disponible en: 〈http://www.injuv.gob.cl/injuv2010/6_encuesta〉. Acceso en: 5 abr. 2011.

LARRAÑAGA, O. Eficiencia y equidad en el sistema de salud chileno. Serie Financiamiento del Desarrollo, n. 49, CEPAL, 1997.

MARÍN, C. Conocimientos, actitudes y prácticas de los gineco-obstetras sobre la anticoncepción hormonal de emergencia (AE). Estudio piloto en San José de Costa Rica, año 2002. Población y Salud en Mesoamérica, v. 1, n. 2, p. 1-9, 2004. Disponible en: «http://redalyc.uaemex.mx/pdf/446/44601201. pdf). Acceso en: 3 abr. 2011.

MARTIN, A. La anticoncepción de emergencia en América Latina y El Caribe. Revista Panameña de Salud Pública, v. 16, n. 6, p. 424-431, 2004. Disponible en: «http://www.scielosp.org/pdf/rpsp/v16n6/23691. pdf>. Acceso en: 3 abr. 2011.

MENG, C.-X.; MARIONS, L.; BYSTRÖM, B.; GEMZELL-DANIELSSON, K. Effects of oral and vaginal administration of levonorgestrel emergency contraception on markers of endometrial receptivity. Human Reproduction, v. 25, n. 4, p. 874-883, 2010.

MIDEPLAN, DIVISIÓN SOCIAL. Encuesta CASEN. Santiago: MIDEPLAN, 2009. Disponible en: «www. mideplan.cl/casen>. Acceso en: 30 mar. 2011.

MOLINA, R.; MOLINA, T.; GONZÁLEZ, E. Madres niñas-adolescentes de 14 años y menos. Un grave problema de salud pública no resuelto en Chile. Revista Médica de Chile, n. 135, p. 79-86, 2007. Disponible en: 〈http://www.scielo.cl/scielo.php?pid=S0034-98872007000100011\&script=sci_arttext〉. Acceso en: 7 abr. 2011.

MORÁN FAÚNDES, J. M. “Índice CLAE” de acceso a la anticoncepción de emergencia. La situación de la anticoncepción de emergencia en América Latina y El Caribe: barreras y facilitadores en la accesibilidad. San José: Consorcio Latinoamericano de Anticoncepción de Emergencia, 2010. Disponible en: 〈http:// www.colectiva-cr.com/Anexo\%2013\%20Indice\%20CLAE\%202010\%20final.pdf〉. Acceso en: 4 abr. 2011.

OYARZÚN, E. Contracepción de emergencia. Estudios Públicos, n. 95, p. 25-41, 2004.

PANTELIDES, E. A; BINSTOCK, G. La fecundidad adolescente en la Argentina al comienzo del siglo XXI. Revista Argentina de Sociología, v. 5, n. 9, p. 24-43, 2007. Disponible en: 〈http://www.scielo.org.ar/ scielo.php?pid=S1669-32482007000200003\&script=sci_arttext>. Acceso en: 11 abr. 2011.

RAYMOND, E. G.; TRUSSELL, J.; POLIS, C. B. Population effect of increased access to emergency contraceptive pills. Obstetrics \& Gynecology, v. 109, n. 1, p. 181-188, 2007.

RODRÍGUEZ, J. Reproducción en la adolescencia: el caso de Chile y sus implicaciones de política. Revista de la CEPAL, v. 86, p. 123-146, ago. 2005.

RODRIGUEZ, J.; DI CESARE, M. Reproducción adolescente y desigualdades en Chile: tendencias, determinantes y opciones de política. Revista de Sociología, n. 23, p. 39-65, 2010. Disponible en: 〈http://www.facso.uchile.cl/publicaciones/sociologia/docs/rev_socio\%20_23/rodriguez_di_cesare. pdf $>$. Acceso en: 7 abr. 2011. 
ROMERO MEZARINA, L. G.; LLANOS ZAVALAGA, L. F.; SALHUANA HUAMAN, J. J.; MAYCA PÉREZ, J.; QUIJANO RONDAN, B. R. Conocimientos y actitudes de médicos gíneco-obstetras de Lima y Callao, sobre los mecanismos de acción y prescripción de la anticoncepción oral de emergencia. Revista Médica Herediana, v. 18, n. 2, p. 92-99, 2007. Disponible en: 〈http://www.scielo.org.pe/scielo.php?script=sci_ arttext\&pid=S1018-130X2007000200006>. Acceso en: 12 abr. 2011.

SCHKOLNIK, S. La fecundidad en América Latina. Serie Seminarios y Conferencias, n. 36, p. 33-47, 2004. Disponible en: 〈http://www.eclac.org/publicaciones/xml/3/22713/LCL2097.pdf〉. Acceso en: 6 abr. 2011.

SOUZA, R. A.; BRANDÃO, E. R. Marcos normativos da anticoncepção de emergência e as dificuldades de sua institucionalização nos serviços públicos de saúde. Physis Revista de Saúde Coletiva, v. 19, n. 4, p. 1067-1086, 2009. Disponible en: «http://www.scielo.br/scielo.php?script=sci_arttext\&pi $d=$ S0103-73312009000400009>. Acceso en: 12 abr. 2011.

SWAHN, M. L.; WESTLUND, P.; HOHANNSSON, E. Effect of postcoital contraception method on the endometrium and the menstrual cycle. Acta Obstetricia et Gynecologica Scandinavica, v. 75, n. 8, p. 738-744, 1996.

VON HERTZEN, H.; PIAGGIO, G., DING, J.; CHEN, J.; SONG, S.; BÁRTFAI, G.; NG, E.; GEMZELL-DANIELSSON, K.; OYUNBILEG, A.; WU, S.; CHENG, W.; LÜDICKE, F.; PRETNAR-DAROVEC, A.; KIRKMAN, R.; MITTAL, S.; KHOMASSURIDZE, A.; APTER, D.; PEREGOUDOV, A. Low dose mifepristone and two regimen of Levonorgestrel for emergency contraception: a Who multicentre randomized trial. Lancet, v. 360, n. 9348, p. 1803-1810, 2002.

YUZPE, A. A.; SMITH, R. P.; RADEMAKER, A. W. A multicenter clinical investigation employing ethinyl estradiol combined with dl-norgestrel as postcoital contraceptive agent. Fertil Steril, v. 37, n. 4, p. 508-513, 1982.

\section{Resumo}

Contraceptivo de emergência no Chile: estruturação da sua demanda em função das variáveis socioeconômicas

Em janeiro de 2010 foi aprovada, no Chile, a Lei n. 20.418 que restabeleceu a entrega do contraceptivo de emergência (pílula do dia seguinte) no serviço público de saúde do país, que tinha sido proibida pelo Tribunal Constitucional, em 2007. Mais de um ano depois da entrada em vigor da Lei, o Ministério da Saúde publicou dados oficiais sobre a entrega deste método contraceptivo para o ano de 2010, o que abre novas possibilidades para entender os níveis de acessibilidade e de demanda. 0 presente trabalho analisa, pela primeira vez, a estrutura desta demanda no Sistema Nacional de Serviços de Saúde, avaliando a incidência de três variáveis socioeconômicas: a condição econômica, a escolaridade e a área de residência. Os resultados mostram que a taxa média de consultas pelo contraceptivo de emergência, entre mulheres de 15 a 44 anos, foi de 2,53 consultas por mil mulheres em idade fértil. Essa taxa corresponde a 4,27, para as adolescentes de 15 a 19 anos, e a 2,1, entre as mulheres de 20 a 44 anos. Os maiores níveis da demanda foram registrados nas comunidades com indicadores socioeconômicos deficientes (comunidades de baixa renda), ou seja, elevadas proporções de população de comunidades de baixo poder aquisitivo, de pessoas sem ensino médio completo e de população residente nas zonas rurais.

Palavras-chave: Contraceptivo de emergência. Pílula do dia seguinte. Chile. Reprodução. Fertilidade. 


\section{Abstract \\ Emergency contraception in Chile: The structure of demand based on socio-economic variables}

In January 2010 Chilean law restored the provision of emergency contraception (morning-after pill) at public health services, after the Constitutional Court had banned its distribution in 2007. More than a year after the law went into force the Ministry of Health published, for the first time, official data on the application of this contraceptive method, a fact that opens up new possibilities for understanding its levels of demand and accessibility. This paper analyzes the demand on the national health system, evaluating the effects of three socioeconomic variables: economic status, educational level and place of residence. The results show that the average number of appointments for emergency contraception in women between the ages of 15 and 44 was 2.53 per 1,000 women. The rate was 4.27 for adolescent girls between ages 15 and 19, and only 2.1 in women aged 20-44. The highest levels of demand were seen in areas with low socio-economic indicators. In other words, a high percentage of the users were from low-income areas, many of them not having finished high school, and a high proportion lived in rural areas.

Keywords: Emergency contraception. Morning-after pill. Chile. Reproduction. Fecundability.

Recebido para publicação em 16/04/2011

Aceito para publicação em 05/06/2011 